\title{
Nurses' perceptions on implementing a task-shifting/sharing strategy for hypertension management in patients with HIV in Nigeria: a group concept mapping study
}

Angela Aifah $^{1 *}$ D, Deborah Onakomaiya ${ }^{1}$, Juliet Iwelunmor ${ }^{2}$, David Oladele ${ }^{3}$, Titilola Gbajabiamila ${ }^{3}$, Chisom Obiezu-Umeh², Ucheoma Nwaozuru², Adesola Z. Musa ${ }^{3}$, Oliver Ezechi ${ }^{3}$ and Gbenga Ogedegbe ${ }^{1}$

\begin{abstract}
Background: People living with HIV (PWH) in Africa have higher burden of cardiovascular diseases (CVD) compared to the general population, probably due to increased burden of hypertension (HTN). In this study, we explored nurses' perceptions of factors that may influence the integration of an evidence-based task-shifting/sharing strategy for hypertension control (TASSH) into routine HIV care in Lagos, Nigeria.

Methods: Using group concept mapping, we examined the perceptions of 22 nurses from HIV clinics in Lagos. Participants responded to a focused prompt on the barriers and facilitators of integrating TASSH into HIV care; next, separate focus groups generated relevant statements on these factors; and statements were then sorted and rated on their importance and feasibility of adoption to create cluster maps of related themes. The statements and cluster maps were categorized according to the Consolidated Framework for Implementation Research (CFIR) domains.

Results: All study participants were women and with 2 to 16 years' experience in the provision of HIV care. From the GCM activities, 81 statements were generated and grouped into 12 themes. The most salient statements reflected the need for ongoing training of HIV nurses in HTN management and challenges in adapting TASSH in HIV clinics. A synthesis of the cluster themes using CFIR showed that most clusters reflected intervention characteristics and inner setting domains. The potential challenges to implementing TASSH included limited hypertension knowledge among HIV nurses and the need for on-going supervision on implementing task-shifting/sharing.

\footnotetext{
* Correspondence: angela.aifah@nyulangone.org

${ }^{1}$ Department of Population Health, New York University School of Medicine, New York, NY, USA

Full list of author information is available at the end of the article
}

(c) The Author(s). 2020 Open Access This article is licensed under a Creative Commons Attribution 4.0 International License, which permits use, sharing, adaptation, distribution and reproduction in any medium or format, as long as you give appropriate credit to the original author(s) and the source, provide a link to the Creative Commons licence, and indicate if changes were made. The images or other third party material in this article are included in the article's Creative Commons licence, unless indicated otherwise in a credit line to the material. If material is not included in the article's Creative Commons licence and your intended use is not permitted by statutory regulation or exceeds the permitted use, you will need to obtain permission directly from the copyright holder. To view a copy of this licence, visit http://creativecommons.org/licenses/by/4.0/ The Creative Commons Public Domain Dedication waiver (http://creativecommons.org/publicdomain/zero/1.0/) applies to the data made available in this article, unless otherwise stated in a credit line to the data. 
(Continued from previous page)

Conclusions: Findings from this study illustrate a variety of opinions regarding the integration of HTN management into HIV care in Nigeria. More importantly, it provides critical, evidence-based support in response to the call to action raised by the 2018 International AIDS Society Conference regarding the need to implement more NCD-HIV integration interventions in low-and middle-income countries through strategies, which enhance human resources. This study provides insight into factors that can facilitate stakeholder engagement in utilizing study results and prioritizing next steps for TASSH integration within HIV care in Nigeria.

Keywords: Nurses' perceptions, Group concept mapping, Integrated care, Hypertension, HIV care, Task-shifting, Tasksharing

\section{Contributions to the literature}

- There is an urgent need for evidence-based, integrated care strategies to address the burden of comorbid noncommunicable diseases (NCDs) in patients with HIV, which has become more pervasive in low- and middle-income countries (LMICs). Stakeholders' perceptions are highly important to better adapt these context-specific strategies.

- We found factors related to hypertension training for HIV clinic nurses and on-going supervision to be potential enablers for integrating hypertension management within HIV clinics.

- The findings contribute to the documented gaps in the literature regarding the enablers and barriers of integrating hypertension into HIV care platforms in LMICs.

\section{Background}

Compared to the general population, people living with HIV (PWH) have higher cardiovascular diseases (CVD) related mortality $[1,2]$, likely due to the increased burden of hypertension (HTN) [3], which affects approximately $14 \%$ of PWH in Africa [4]. Based on the Global Burden of Disease report, Nigeria had an increase in mortality from 1990 to 2015 among adults with uncontrolled HTN [5]. Because Nigeria still grapples with a stubbornly unrelenting burden of HIV [6] and a shortage in healthcare workers [7], there is an urgent need for evidence-based strategies targeted at HTN control among PWH, particularly strategies which also address the shortage of healthcare workers.

\section{Addressing the health worker shortage and HTN control in Nigeria through task-shifting/sharing}

Similar to other low- and middle-income countries (LMICs), the acute shortage of healthcare workers is a major barrier to HTN control in Nigeria, which has only 3 physicians per 10,000 population [8]. In particular, this shortage of healthcare workers limits Nigeria's capacity to control hypertension at the primary care level, where the majority of PWH receive care. To address healthcare human resource challenges as well as the increasing disease burdens within the country, Nigeria's Ministry of Health $(\mathrm{MOH})$ developed a Task-shifting and Tasksharing Policy for Essential Health Care Services in 2014 with a focus on meeting these challenges at all levels of care [9]. A proven and practical model for improving the management of communicable and non-communicable diseases [10], task-shifting/sharing emphasizes the rational distribution of skills from physicians to underutilized, non-physicians such as nurses and community health workers [7]. While previous studies in Africa have successfully implemented a task-shifting/sharing approach for individual diseases like HIV [11] and hypertension [12], a limited number examine the approach for integrated chronic disease management [13]. Although, the current task-shifting/sharing policy in Nigeria focuses on priority areas such as family and reproductive health, HIV, tuberculosis, and malaria, there is currently no evidence of the policy's implementation in the integration of HTN management and control into HIV care.

\section{Adapting an evidence-based task-shifting/sharing strategy for hypertension control}

In the case of CVD management, task-shifting/sharing has been shown to be an effective strategy in LMIC settings overburdened with healthcare workforce shortages and the increasing prevalence of non-communicable diseases (NCDs) [10]. For example, in a cluster randomized clinical trial of 32 health centers in Ghana, Ogedegbe et al. [7] noted that the addition of a task-shifting strategy for hypertension control or TASSH delivered by trained community health nurses led to a $34 \%$ greater reduction in systolic BP than the provision of health insurance coverage alone [7]. Based on the WHO CVD Risk Package [14], components of the TASSH intervention include (1) CVD management clinical decision support using validated WHO risk charts, (2) lifestyle counseling for patients, and (3) medication titration (either increasing a dose or adding a second medication) and patient referral to physicians for the management of 
complicated HTN [7]. Nurses in the intervention arm were trained in each of these components during yearly training sessions for the study duration [7]. While TASSH has shown to be successful in controlling hypertension in Ghana, the intervention has yet to be applied in integrating hypertension into the HIV care cascade.

\section{Prioritizing the need to integrate hypertension control into HIV care in Nigeria}

With access to highly active antiretroviral treatment (HAART) in LMICs leading to increased survival [2], people living with HIV (PWH) are at an increased risk for NCDs like CVD [15]. The 2018 International AIDS Society Conference acknowledged the growing burden of comorbid NCDs in PWH and proposed an integrated model of care whereby management of NCDs is integrated into HIV chronic care platforms as a cost-effective strategy $[15,16]$. Researchers at the meeting also expressed an urgent need for more evidence on implementing integrated HIV-NCD care in LMICs with a critical shortage of health workforce [15]. Based on the successful implementation of TASSH in Ghana [7] along with the similarity in healthcare systems of both countries, the current study explores the perceptions of HIV clinic nurses on the barriers and facilitators of integrating TASSH into routine HIV care for the management of HTN in PWH in Lagos, Nigeria. This information enabled the research team to evaluate the feasibility of implementing TASSH as an integrated model for the management of HTN in HIV clinics for an NIH grant submission, which has since been awarded.

\section{Methods and data analysis}

\section{Study setting and recruitment}

This study took place at the Nigerian Institute of Medical Research (NIMR) - a research arm of the Federal Ministry of Health, Nigeria. NIMR offers a wide spectrum of public health services and clinical research in HIV, tuberculosis, malaria, neglected tropical diseases, maternal and reproductive health, and NCDs among others. It has a comprehensive HIV treatment center with over 25,000 PWH cumulatively enrolled in care since 2004. A total of 22 HIV clinic nurses were recruited, from 20 HIV clinics in Lagos, to participate in the study through convenience sampling wherein supervisors selected nurses based on staffing availability. The $20 \mathrm{HIV}$ clinics were a subset of the clinics that will be selected for the proposed intervention. In addition to being representative of the $20 \mathrm{HIV}$ clinics, all of the HIV nurses participated in the brainstorming as well as the sorting and rating activities while 18 of the 22 nurses participated in the group concept mapping (GCM) discussion activity. The rationale for the sample size was based on similar studies using GCM strategy [17]. All participants provided written informed consent and the study was approved by NIMR's Institutional Review Board.

\section{Study objective and design}

To better understand HIV nurses' perceptions on TASSH, we employed GCM methodology. GCM uses a mixedmethods participatory approach to stakeholder engagement, in a six-step process (preparation, generation, structuring, representation, interpretation, and utilization) to assess the perceptions of participants on a specific topic by generating illustrative conceptual frameworks of the target group's views $[18,19]$. For this study, HIV clinic nurses were engaged in GCM activities to elicit their thoughts on an integrated HIVNCD model using a task-shifting/sharing strategy for the management of hypertension in PWH. GCM analyses were conducted using the Concept Systems Global Max software (Concept Systems, Inc., Ithaca, NY) [18]. Figure 1 depicts how the GCM methodology was adapted for the purposes of this study in the following stages: brainstorming, sorting and rating, and GCM data analysis and final group discussion. At the first GCM group meeting, a discussion took place on the aims and outcomes of the Ghana TASSH intervention study to address any questions participants had about the intervention. Data was collected in March 2019.

\section{Brainstorming stage}

The nurses provided succinct statements in response to the following prompt: "In order for HIV clinic nurses to successfully adopt a task-shifting/sharing strategy for hypertension control among people living with HIV, they need...." Responses were manually entered into the Concept Systems software to generate a list of statements that reflects each nurse's response by creating individual profiles for each nurse with a unique ID code. This process yielded a final set of statements which were randomly sorted and printed out on separate sorting cards for group discussion. Nurses also responded to a brief demographic survey on their applicable HIV work experience.

\section{Sorting and rating stage}

The nurses participated in two separate focus group sessions [led by AA and DO], where they worked in pairs to sort the final list of statements into grouped piles that made sense to them and based on their current practice within the HIV clinics. Participants were instructed to create as many piles as needed while making sure there were not too many or too few piles. After sorting the statements, they then rated each statement on their perceived importance [on a 3-point scale, with 1 being "important" and 3 being "very important"] and potential feasibility [on a 4-point Likert scale, with 1 being "not feasible" and 4 being "extremely feasible"] concerning the proposed intervention [TASSH]. 


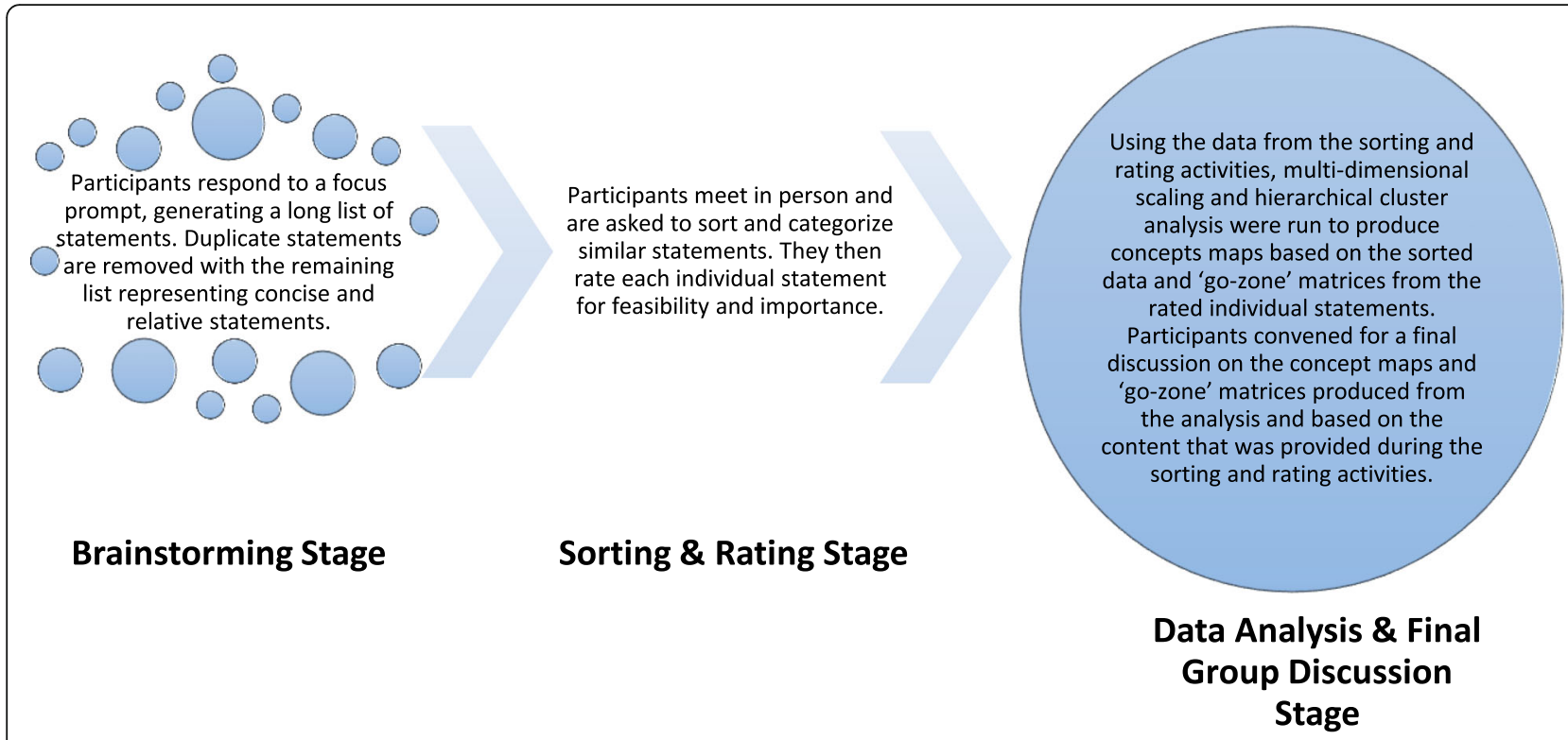

Fig. 1 GCM stages

\section{Data analysis}

After sorting and rating, the final statements were entered into the Concept Mapping software, which uses multidimensional scaling (MDS) analysis to graphically show points representing statements that were frequently sorted together being more proximal to each other and infrequently sorted statements being further apart [20]. MDS produces several visual outputs including a point map, cluster map, and go-zone rating-all of which are shared with the participants to discuss their interpretability and to come to an agreement on the representativeness of the raw data with the outputs $[19,21]$. For example, the "cluster map" output shows how the statements generated, represented by points, were grouped by participants [21]. In this study, the MDS yielded two viable cluster maps representing 8 and 12 cluster groupings of statements.

\section{Interpreting the GCM maps through final group discussion}

Following the MDS analysis, another focus group with a subset of the nurses (18 total) was convened to provide them feedback on the two cluster maps and the final cluster map groupings and discuss their views. For this purpose, we printed out the cluster maps and shared the analytical outputs with the nurses. The nurses were asked to vote on which cluster, i.e., choosing between the 8 or 12 cluster grouping, was most representative of their perspectives of TASSH. They were also asked to provide feedback on potential adaptations to TASSH based on their interpretation of the cluster maps. The nurses were also presented with Go-Zone matrices which show sets of rated statements in the cluster maps, where each statement is assigned coordinates based on mean rating on $x$ - and $y$-axis. The Go-Zone creates four quadrants based on ratings of the statements (above or below the mean) for its importance and feasibility of integrating it into the proposed intervention [TASSH]. The Go-Zone creates the following zones: high importance-high feasibility (green zone), high importance-low feasibility (yellow zone), low importance-high feasibility (orange zone), and low importance-low feasibility (gray zone). The high-high quadrant (i.e., high importance and high feasibility) is typically considered the Go-Zone because it includes the ideas rated most highly for both criteria, which in this case, is the importance and feasibility of the rated statement for integrating hypertension management into HIV care via a task-shifting/sharing strategy. At the conclusion of this focus group, the nurses also provided additional feedback on specific challenges for integrating an intervention like TASSH into HIV care. Inductive thematic analysis was conducted for this final feedback.

\section{Mapping the clustered themes to CFIR}

Following the group GCM activities, we used the Consolidated Framework of Implementation Research (CFIR) to map the 12 clusters and further assess how they could be tailored for the implementation of the intervention. CFIR also provides a practical guide for evaluating stakeholders' perceptions on the feasibility and importance of implementing an evidence-based program, in this case, TASSH. Developed by Damschroder and colleagues, CFIR provides an overarching typology for the systematic assessment of factors, which may influence an intervention's implementation and effectiveness [22, 23]. Consisting of five domains (intervention characteristics, outer setting, inner setting, characteristics of the individuals involved, and the process of 
implementation), each with their related constructs, CFIR has been used as a rapid-cycle evaluation guide of stakeholder's perceptions on the implementation of a healthcare intervention [23].

\section{Results}

Applying GCM, nurses generated a total of 125 statements, which were reviewed by two members of the research team (AA and DO) to remove redundant statements and statements not relevant to the prompt, leaving a final set of 81 statements. Figure 2 represents the point map of the 81 statements, depicting the varied spread and relative closeness of the statements. In the following sections, we provide the findings on participant's TASSH-related demographic data, results from the GCM activities, the mapping of the 12 clusters to the CFIR domains, and feedback on specific challenges for integrating TASSH.

\section{Participants' TASSH-related demographic data}

All study participants were women, with 2 to 16 years' experience in the provision of HIV care. Initial group discussions with participants showed that they were familiar with the task-shifting/sharing approach but had not participated in a task-shifting/sharing intervention for integrating hypertension management and control into HIV treatment at their local clinics. The nurses had worked in HIV care for an average of 6 years, and they care for approximately 79 patients per day, of which about 41 are PWH. Regarding the essential duties of the nurses specifically related to components of the proposed TASSH intervention, $60 \%$ routinely perform finger prick glucose test; $67 \%$ conduct urine dipstick, with approximately $94 \%$ engaged in patient education and counseling on lifestyle modification.

\section{GCM activities' output}

As noted in Fig. 3, the nurses selected a 12-cluster map which they believed best characterized their understanding of the key factors that may facilitate or hinder the integration of hypertension management into HIV care using a task-shifting/sharing strategy. For GCM, and through the MDS analysis, a "stress value" of the point map is typically generated to determine how well the MDS solution maps to the inputted data and indicates a goodness of fit [21]. In this study, the stress value for the cluster maps was 0.29 demonstrating overall good fit. The representative statements for each of the 12 clusters along with their corresponding number are shown in Table 1.

Figure 4 shows the Go-Zone ratings generated for all 81 statements and the 12 cluster map. Five statements were rated high for importance and feasibility of integrating hypertension management into HIV care. These include "Ensure regular monitoring of BP at every clinic visit" (statement 5); "Provision of counselling to the patients" (statement 17); "Monitor vital signs regularly" (statement 18); "Re-educating patients on importance of diet, rest, and adherence in the management of HIV/ Hypertension" (statement 26); and "Encouraging patient on the importance of adherence to drug regimen to prevent relapse in healthcare" (statement 34). Conversely, the statement rated the lowest for importance and feasibility was "Ensuring that each patient has their BP

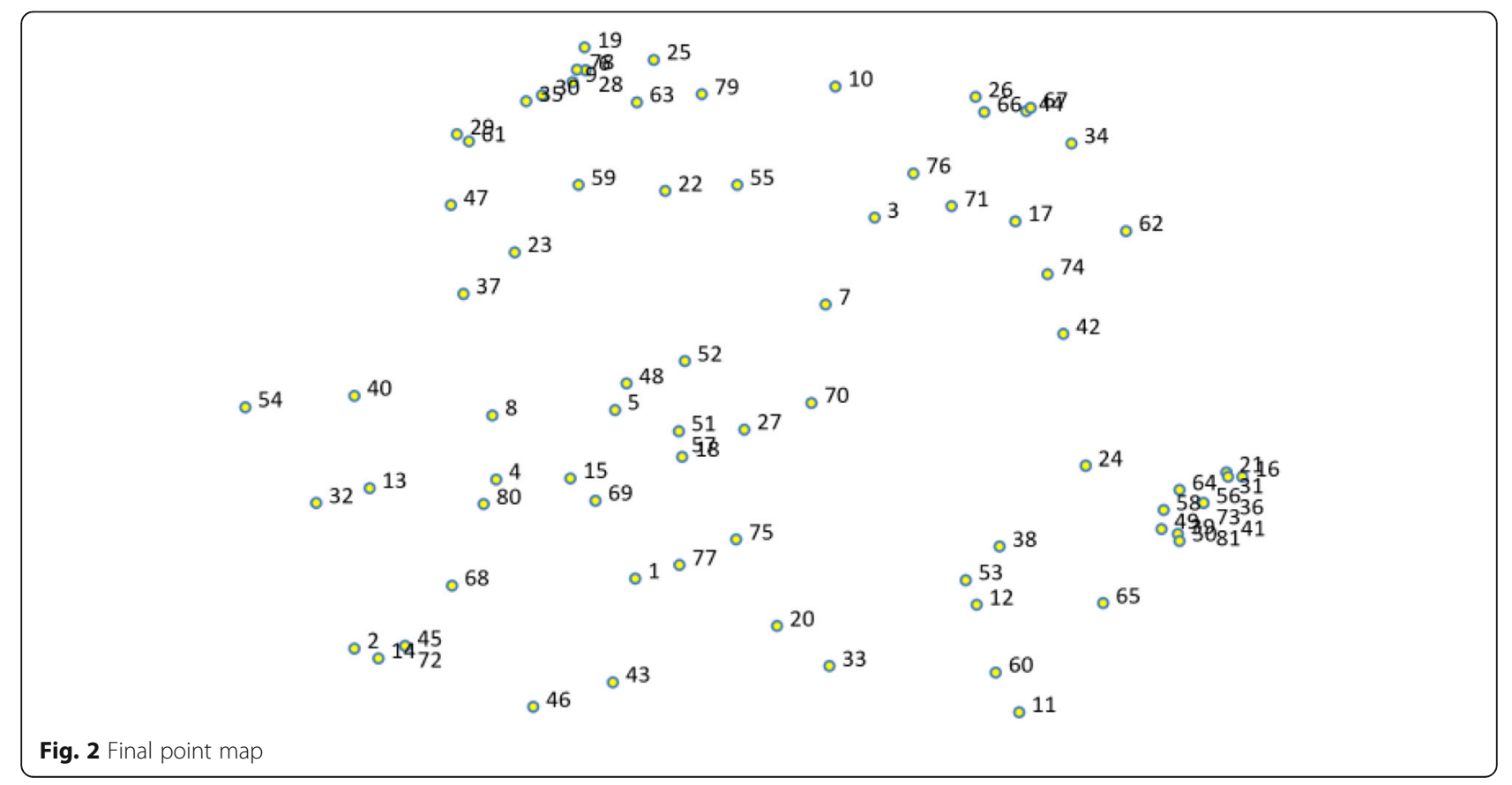




\section{Cluster Map}

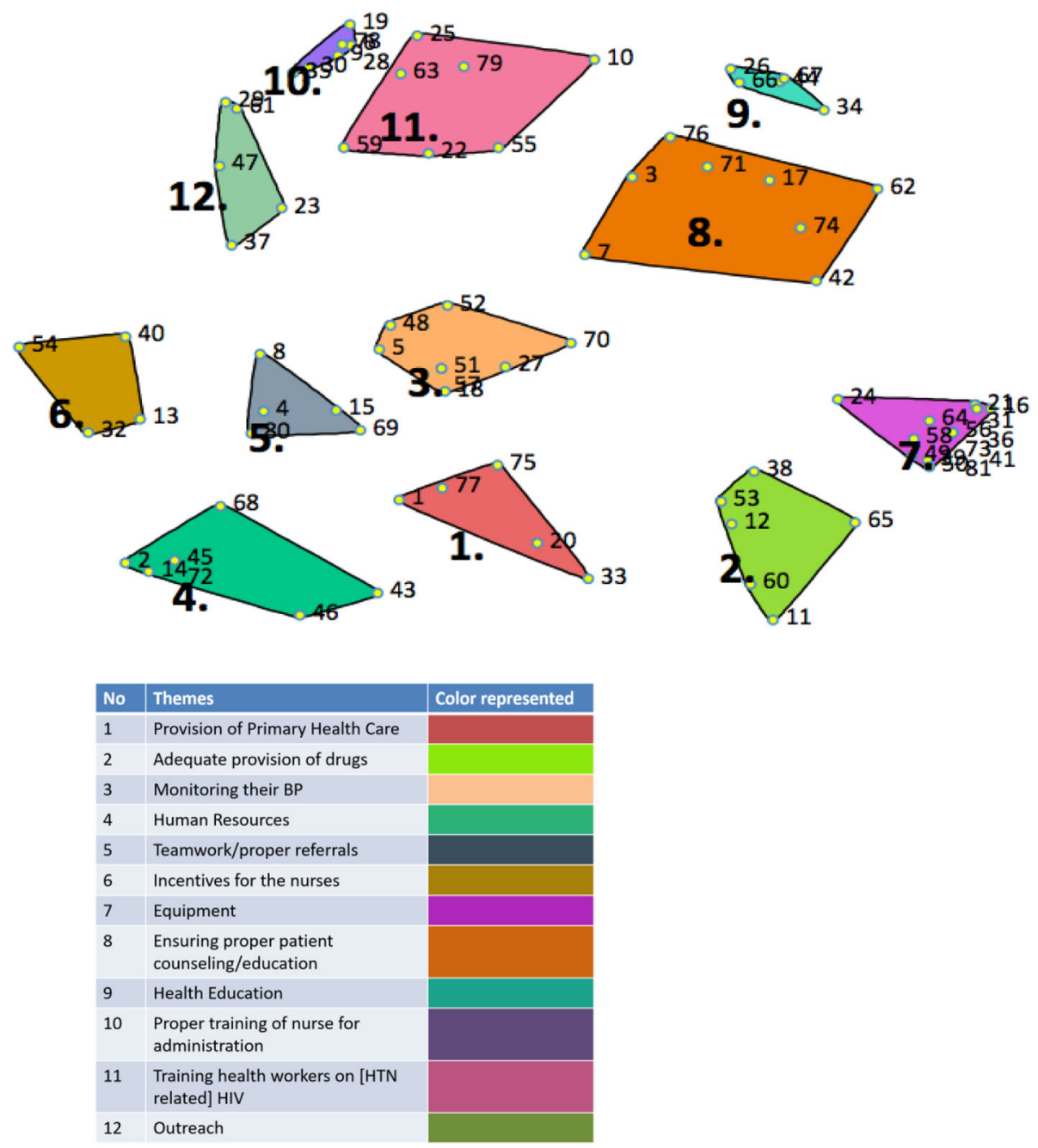

Fig. 3 Cluster map with corresponding legend for the 12 themes

apparatus to monitor their blood pressure at regular intervals and comes to the hospital in case there is any rise in blood pressure" (statement 42).

\section{The 12 cluster themes and the CFIR domains}

Based on the discussions with the nurses and their applicability to the study, we mapped the themes or clusters to four domains of CFIR-i.e., inner settings, outer settings, intervention characteristics, and characteristics of the individuals involved. The fifth domain, implementation process, was not applicable for the purpose of this study. Table 2 shows the CFIR domains and the corresponding theme or cluster. The majority of the 12 cluster themes selected by nurses were considered by the research team to be most applicable to the inner setting and intervention characteristics domains. While the individuals involved domain only had one cluster theme that seemed most relevant.

\section{Feedback on specific challenges for integration}

After the GCM discussions and as part of their closing remarks at the final group meeting, the nurses provided additional feedback on content specific barriers that might hinder the integration of hypertension management into HIV care in their clinics. The barriers identified were divided into the following categories: nurses' perspectives on work-related challenges and nurses' perspectives on patient barriers. Nurses' perspectives on work-related challenges included the shortage of nurses at primary healthcare centers; work ethics of health 
Table 1 Twelve clusters with corresponding statements

\begin{tabular}{|c|c|}
\hline Themes & Statements \\
\hline \multirow[t]{5}{*}{ 1. Provision of primary health care } & 1. Their closeness to the facility is considered too. \\
\hline & 20. Availability of drugs at an affordable price for patient consumption. \\
\hline & $\begin{array}{l}\text { 33. Restructuring the PHC setting in such a way that the nurse is officially allowed to see the sick when the } \\
\text { doctor is not around or very busy especially at the outpatient clinic and emergency clinic. }\end{array}$ \\
\hline & 75. Making ART clinic a one-stop clinic for PLHIV/hypertension to reduce waiting time. \\
\hline & 77. Prompt linkage of hypertensive clients to physician and other relevant facilities for further management. \\
\hline \multirow[t]{6}{*}{ 2. Adequate provision of drugs } & 11. Financial support. \\
\hline & 12. Provision of primary health care. \\
\hline & 38. Working environment should be more conducive for the health worker clients. \\
\hline & 53. Conducive work environment. \\
\hline & 60. Provision of subsidized anti-hypertensive drugs. \\
\hline & 65. Drugs for management of hypertension should be available in the HIV clinic. \\
\hline \multirow[t]{8}{*}{ 3. Monitoring their $\mathrm{BP}$} & 5. Ensure regular monitoring of BP at every clinic visit. \\
\hline & 18. Monitor vital sign regularly. \\
\hline & 27. Proper clinic standard operating procedure (SOP). \\
\hline & 48. Proper follow-up strategies on clients. \\
\hline & 51. Monitor intake and output chart. \\
\hline & 52. Great nurse/patients relationship \\
\hline & 57. Monitoring of blood pressure. \\
\hline & 70. Proper supervision of standard operating procedure (SOP). \\
\hline \multirow[t]{7}{*}{ 4. Human resources } & 2. Employ more nurses. \\
\hline & 14. Availability of human resources. \\
\hline & 43. The PHC organogram should be revisited to put the nurse in their proper position. \\
\hline & 45. Human resources-more nurses. \\
\hline & 46. More nurses to be employed into the PHC setting. \\
\hline & 68. Relegation of power-let people take responsibility. \\
\hline & 72. Adequate man power. \\
\hline \multirow[t]{5}{*}{ 5. Teamwork/proper referrals } & 4. Ensuring that each clinic, i.e., both HIV and medical outpatient clinic (hypertension) falls on the same visit. \\
\hline & 8. Supervision of staff on providing care. \\
\hline & 15. Aligning both clinic appointments to fall on the same day. \\
\hline & $\begin{array}{l}\text { 69. Providing measures in improving their health care, i.e., the clinic should be interwoven (the appointment } \\
\text { should always be the same date) to avoid excessive stress. }\end{array}$ \\
\hline & 80. Effective teamwork. \\
\hline \multirow[t]{4}{*}{ 6. Incentives for the nurses } & 13. Encouragement of work delegation. \\
\hline & 32. Consideration of the number of nurses in a clinic \\
\hline & $\begin{array}{l}\text { 40. There should be clarity on which task is shifting to who, to avoid unnecessary rivalry among health } \\
\text { workers }\end{array}$ \\
\hline & 54. Incentive for the nurses. \\
\hline \multirow[t]{7}{*}{ 7. Equipment } & 16. More working tools like protective measure-gloves, apron, boots, cap and face mask, and testing kits. \\
\hline & 21. Provision of blood pressure apparatus. \\
\hline & 24. Blood pressure monitoring manual. \\
\hline & 31. Provision of functioning sphygmomanometer at the clinic always. \\
\hline & 36. Adequate provision of materials/equipment. \\
\hline & 39. Government should be ready to supply and provide everything needed for \\
\hline & 41. We need materials or instrument to manage the HIV/hypertension patient. \\
\hline
\end{tabular}


Table 1 Twelve clusters with corresponding statements (Continued)

\begin{tabular}{|c|c|}
\hline Themes & Statements \\
\hline \multirow{15}{*}{$\begin{array}{l}\text { 8. Ensuring proper patient } \\
\text { counseling/education }\end{array}$} & 49. Provision of HIV test kit and drugs. \\
\hline & 50. Provision of facilities needed. \\
\hline & 56. Available equipment to work with. \\
\hline & 58. Weighting scales (SECA) with height. \\
\hline & 64. Enhance current medical equipment \\
\hline & 73. Regular supply of working tools and necessary materials. \\
\hline & 81. Provision of better equipment to work with. \\
\hline & 3. Counseling of patient for follow-up. \\
\hline & 7. Proper follow-up and non-abrupt stoppage of BP drug. \\
\hline & 17. Provision of counseling to the patients. \\
\hline & 42. Ensuring that each patient has their BP apparatus to monitor their blood. \\
\hline & 62. Ensuring daily use of anti-hypertensive drug. \\
\hline & 71. Proper health education on diet and lifestyle. \\
\hline & $\begin{array}{l}\text { 74. Encourage clients to have personal BP apparatus for their home use, in order to identify BP and report } \\
\text { before appointment day if necessary. }\end{array}$ \\
\hline & 76. Proper health education on hypertension with people living with HIV during each visit to the clinic. \\
\hline \multirow[t]{5}{*}{ 9. Health education } & $\begin{array}{l}\text { 26. Re-educating patients on importance of diet, rest and adherence in the management of HIV/ } \\
\text { hypertension. }\end{array}$ \\
\hline & 34. Encouraging patient on the importance of adherence to drug regimen to prevent relapse in healthcare. \\
\hline & $\begin{array}{l}\text { 44. Stressing the do's and don'ts of people living with HIV and hypertension, i.e., smoking, drinking of alcohol, } \\
\text { and use of protective materials during sexual intercourse. }\end{array}$ \\
\hline & 66. We need to give them quality counseling. \\
\hline & 67. Intensified health education on hypertension with people living with HIV on clinic days. \\
\hline \multirow{7}{*}{$\begin{array}{l}\text { 10. Proper training of nurses for } \\
\text { administration }\end{array}$} & 6. Training for nurse to improve their knowledge on hypertension. \\
\hline & 9. Nurses need better understanding of the case. \\
\hline & 19. Proper training of nurses for administration. \\
\hline & 28. Training to nurses on how to provide psycho-social support to patients. \\
\hline & 30. Training of health workers on the update of HIV drugs. \\
\hline & 35. Constant continuous communication in the form of training and re- training for nurses. \\
\hline & 78. We need proper training to be able to manage the patients. \\
\hline \multirow{7}{*}{$\begin{array}{l}\text { 11. Training for health workers on } \\
\text { [HTN related] HIV }\end{array}$} & 10. Personal training on adherence counseling on lifestyle modification, medication, and diet. \\
\hline & 22. We need to know the patient's problems. \\
\hline & 25. Training on screening patient with cardiovascular risk. \\
\hline & 55. Provide detailed and good understanding of task-shifting strategies for hypertension control. \\
\hline & 59. Constant review of training workshop of nurses and more health workers on ground. \\
\hline & 63. Adequate knowledge of anti-retrovirals that can affect blood pressure. \\
\hline & 79. Knowledge on the treatment of hypertension. \\
\hline \multirow[t]{5}{*}{ 12. Outreach } & 23. Early detection of people living with hypertension. \\
\hline & 29. Sensitization. \\
\hline & 37. Prioritizing patient health care according to their health care needs. \\
\hline & $\begin{array}{l}\text { 47. There should be good advocacy, communication between health workers and the community to know } \\
\text { prevention and precaution and how to go about it. }\end{array}$ \\
\hline & 61. Awareness—sensitize the environment. \\
\hline
\end{tabular}




\section{Go Zone}

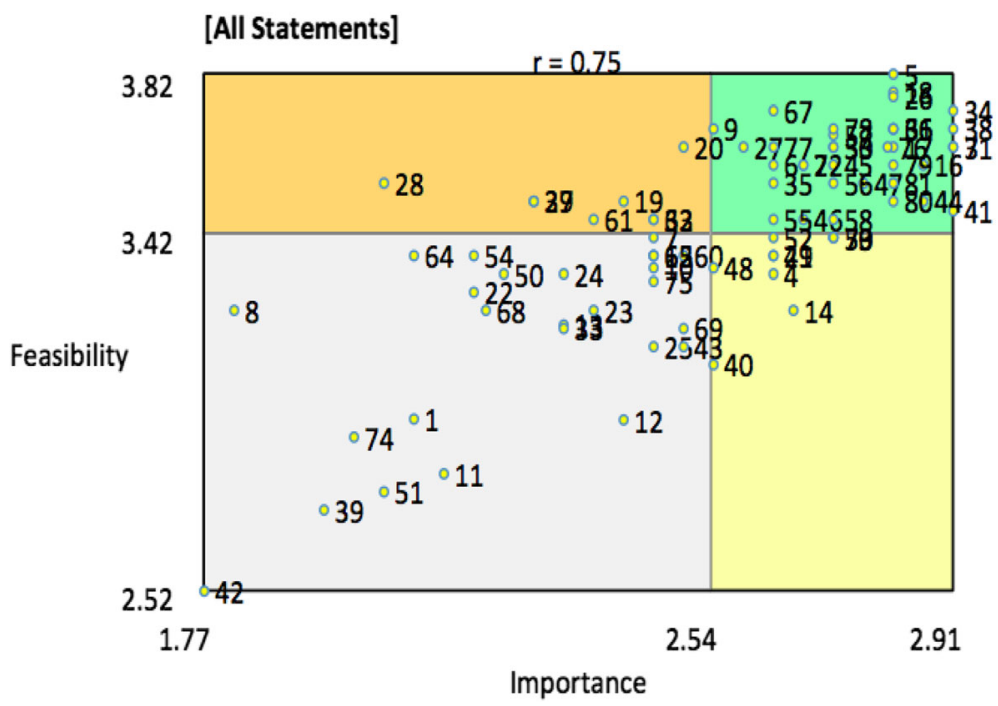

Fig. 4 Feasibility and importance Go-Zone

workers (including nurses); limited knowledge of hypertension, CVD risk assessment, and prescribing hypertension medication; and inadequate provision of tools/ resources for hypertension management. Regarding nurses' perspectives on patient barriers, the following were highlighted: patients' limited knowledge of hypertension and self-management; the inability of patients to prioritize payments for anti-hypertensive medication in addition to HIV medication; and high medication cost.

\section{Discussion}

To our knowledge, this is the first study to use a rigorous mixed-methods GCM methodology to explore the perceptions of HIV clinic nurses on the factors that may facilitate or hinder the integration of hypertension management into the HIV care using an evidence-based task-shifting/sharing strategy for hypertension control. We identified 81 statements, which were grouped into 12 clusters representing key factors HIV nurses chose as most comprehensive or fitting for implementing a taskshifting/sharing strategy to integrate hypertension management into HIV care.

As noted in the cluster map and based on other studies using concept mapping [24], the points or statements which are closer denote those themes which the MDS analysis found to be more closely related than those points which are farther apart. The same logic applies to the clusters themselves, i.e., those in close proximity are

Table 2 CFIR domains and the associated clusters

\begin{tabular}{|c|c|}
\hline CFIR domains & Associated cluster themes \\
\hline Inner setting: features of the healthcare system within which TASSH is implemented & $\begin{array}{l}\text { - Human resources } \\
\text { - Teamwork/proper referrals } \\
\text { - Equipment } \\
\text { - Outreach }\end{array}$ \\
\hline $\begin{array}{l}\text { Outer setting: the external context to the settings where TASSH is being } \\
\text { implemented }\end{array}$ & $\begin{array}{l}\text { - Incentives for nurses } \\
\text { - Provision of primary health care access } \\
\text { - Adequate provision of drugs }\end{array}$ \\
\hline Intervention characteristics: features of the proposed intervention & $\begin{array}{l}\text { - Training health workers on management of [HTN related] } \\
\text { HIV } \\
\text { - Ensuring proper patient counseling/education } \\
\text { - Health education } \\
\text { - Proper training of nurses on components of the intervention }\end{array}$ \\
\hline Individuals involved: characteristics of participants involved in the implementation & - Monitoring their [patient] BP \\
\hline
\end{tabular}


conceptually related in comparison to those farther apart. In terms of the size or shape of the clusters, Schell and Luke [24] note that typically smaller, denser clusters represent concepts or statements which the HIV nurses believed to be more acceptable or compatible; while the larger clusters denote more dispersed ideas. Interestingly, statements represented in clusters 7 ("equipment"), 10 ("proper training of nurses for administration"), and 9 ("health Education") were proximal to one another, showing that the statements for each cluster were closely related to each other. These three clusters were also among the smaller, denser groupings-meaning that HIV nurses found these statements to be more acceptable for implementing a task-shifting/sharing strategy for hypertension management. Additionally, the stress value of 0.29 indicates that the final cluster was representative of the nurses' perspectives. For GCM, a lower stress value indicates a better fit and reflects a stronger relationship between optimal and actual configurations [21]. Other studies have used a value of 0.35 or greater to indicate a higher stress value, i.e., a greater discrepancy between the inputted data and the generated concept map [25]. Our findings are in line with those of other studies that evaluated taskshifting/sharing strategy for hypertension management [7, $12,17,26]$. Blackstone et al. [26] also noted that proper training of nurses was particularly important for sustaining a task-shifting/sharing strategy for hypertension management.

This study extends our previous work on the capabilities, opportunities, and motivations of HIV clinics in Lagos State's practice capacity to integrate hypertension management into HIV care [27], by specifically focusing on the perspectives of the HIV clinic nurses on the barriers and facilitators of implementing a task-shifting/ sharing intervention. The application of GCM noted that for HIV nurses, implementing TASSH as part of the routine care was not only feasible but also regarded as highly important to adequately address the burden of hypertension among PWH. Furthermore, the low stress value from the concept mapping analysis shows congruency in the sorted and rated statements by the HIV nurses and the generated maps - validating that this mixed-methods approach adequately represented the perceptions of the HIV nurses. Other GCM studies have also noted similar findings regarding the representativeness of a lower stress value for the final model with the statements generated by participants [26, 28].

Findings from our study underscore the importance of developing nurse-based strategies to integrate hypertension management into HIV care given the limited availability of evidence supporting context-specific strategies in Africa [29]. A recent review by Vorkoper and colleagues noted that implementation science research will be critical in efforts to integrate evidence-based interventions into existing HIV chronic care platforms given the emphasis on context-appropriate approaches for sustained health interventions [29]. One such example of a flexible, adaptable implementation science framework is CFIR. The application of CFIR to the 12 clusters or themes chosen by the nurses is a useful approach for evaluating which factors or domains are most relevant for an intervention that is being tailored to a specific context. While all five CFIR domains are most commonly used to understand the context within which evidence-based strategies are implemented [23], in our study, the domain(s) which stood out the most, i.e., those associated with the majority of the themes were inner settings and intervention characteristics, with each matched to 4 of the 12 themes. The fact that most clusters were more applicable to a few CFIR domains as opposed to all, points to the need to consider the specific contextual factors which may have more implications on the implementation and/or adaptation of an intervention like TASSH in a different setting, particularly for integrated care platforms. Although we are not aware if other studies have applied CFIR when analyzing stakeholder's perceptions of task-shifting/sharing strategy for hypertension management in PWH in LMICs, our findings underscore the applicability of using CFIR as an analytical guide for implementing and adapting stakeholder's perceptions of evidence-based interventions.

\section{Limitations and strengths}

There were a few limitations. First, we only sampled 22 nurses, most of whom were staff nurses, but did not include other allied health workers [e.g., Community Health Extension Workers] who might also provide care in HIV clinics in Nigeria. Such a small sample size along with the lack of variety in HIV clinic health workers limits the generalizability of the study findings. Despite this limitation, an asset of the study is the inclusion of participants from HIV clinics where the proposed intervention will be implemented. Second, the concept mapping exercise was labor intensive and required several hours of focus and participation by the nurses over the course of 2 days. This might have prevented more nurses from participating because of work demands at their various health facilities. Third, not all the nurses from the first session completed the concept mapping exercise from the second session (though all nurses at the concept mapping session were part of the first session). While it would have been ideal that all of the HIV clinic nurses participated in all of the GCM related activities and sessions, an advantage of GCM is that it is not a requirement to have every single person participate in each activity [30]. Finally, the mapping of the CFIR domains to the 12 cluster themes did not follow a specific analytical approach but was rather based on a simple synthesis of the themes based on the feedback from 
the HIV nurses. A more thorough content analysis using qualitative methods may have provided additional insight on the applicability of CFIR to the 12 clusters. Despite these limitations, the use of concept mapping supports the application of novel, mixed methods approaches for engaging stakeholders in LMICs who are primarily responsible for adapting task-shifting/sharing strategies.

\section{Implications}

Given the increasing need to address NCDs among PWH in regions with shortages in healthcare workers, interventions tailored to address the dual disease burden while simultaneously enhancing the capacity of key healthcare professionals are of most importance. Despite the establishment of a task-shifting/sharing policy and the prevalence of hypertension among PWHs, Nigeria has yet to institute the approach for integrating NCDs like hypertension into the HIV care continuum. The current study provides contextual evidence of potential barriers and facilitators for implementing a proven task-shifting/sharing intervention for hypertension management within the Lagos State primary healthcare system. Notably, the findings point to factors such as additional health education, human resources, training nurses on hypertension-related HIV treatment, and proper patient counseling or education, which may influence the implementation of a task-shifting/sharing intervention for hypertension management. For an intervention like TASSH to be successfully adopted as part of the routine care offered, there must be targeted consideration for incorporating these factors for integrating hypertension care while simultaneously addressing healthcare worker shortages in HIV clinics in Lagos, Nigeria. Given the burden of comorbid HIV and NCDs in LMICs, future research should consider the feasibility of scaling up task-shifting/ sharing strategy for integrating hypertension management into the HIV care cascade across impacted countries and regions.

\section{Conclusion}

Engaging and understanding stakeholders' perceptions of feasibility of an evidence-based intervention beyond its initial implementation, can provide critical insights for developing sustainable programs in LMICs [17]. Nurses play a major role in the implementation of task-shifting strategies, particularly when addressing integrated care platforms for comorbid HIV and hypertension. For these reasons and more, this study provides critical, evidencebased support in response to the call to action raised by the 2018 International AIDS Society Conference regarding the need to implement more NCD-HIV integration interventions in LMICs through strategies that enhance human resources. As non-communicable diseases become more pervasive among PWH in LMICs still overburdened with communicable diseases and shortages in healthcare workers, context-specific integrated care platforms will be critical in addressing dual disease burden, particularly among PWH and comorbid NCDs. Findings from our study provide highly relevant and emergent evidence in support of the exploration of key stakeholders' perceptions on the feasibility and importance of implementing a task-shifting/sharing strategy for the integration of hypertension management into HIV care.

\section{Abbreviations \\ NIMR: Nigerian Institute of Medical Research; HAART: Highly active antiretroviral therapy; GCM: Group concept mapping; WHO: World Health Organization; CFIR: Consolidated Framework for Implementation Research; CVD: Cardiovascular disease; MOH: Ministry of Health; RCT: Randomized controlled trial; HTN: Hypertension; TASSH: Task-shifting strategy for hypertension control; HIV: Human immunodeficiency viruses; AIDS: Acquired immunodeficiency syndrome; PWH: People living with HIV; NCDs: Non- communicable diseases; LMICs: Low- and middle-income countries; AA: Angela Aifah; DO: Deborah Onakomaiya; Jl: Juliet Iwelunmor; DAO: David Oladele; CO: Chisom Obiezu-Umeh; UN: Ucheoma Nwaozuru; TG: Titilola Gbajabiamila; AM: Adesola Z. Musa; OE: Oliver Ezechi; GO: Gbenga Ogedegbe}

\section{Acknowledgements}

The authors are especially grateful to the key stakeholders, the HIV clinic nurses, who provided invaluable input and generously gave of their time for this study. The authors would also like to extend our gratitude to the HIV clinic administrators and supervisors for their assistance in engaging and granting the nurses the time to participate in the study.

\section{Authors' contributions}

GO conceived the idea for the pilot study. AA developed a protocol for the study. OE and DAO secured IRB approval for the study and recruited the nurses. AA and DO conducted the GCM exercise with nurses and analyzed the data under supervision from JI. UN and CO assisted AA with collecting the initial data from the nurses. DAO and OE provided guidance for interpreting the data provided by the nurses. AA, DO, GO, and Jl supervised and validated the background, methods, and results. GO validated the discussion. AA and DO drafted the full manuscript. CO, UN, TG, and AM reviewed drafts and provided written feedback. GO, JI, DAO, and OE edited the paper for critical content. All authors contributed substantially to the preparation of this manuscript. The authors read and approved the final manuscript.

\section{Funding}

Research reported in this publication was supported by the National Heart, Lung, And Blood Institute of the National Institutes of Health under Award Number R01HL147811. The content is solely the responsibility of the authors and does not necessarily represent the official views of the National Institutes of Health. Drs. Gbenga Ogedegbe, Juliet Iwelunmor, and Oliver Ezechi are supported by a grant received from the NIH R01 HL147811-01A1. Dr. Angela Aifah was supported by grants received from the NIH R01 HL147811-01A1 and T32HL129953.

\section{Availability of data and materials}

All data generated or analyzed during this study are included in this published article.

\section{Ethics approval and consent to participate}

All procedures were in accordance with the ethical standards of the responsible committee on human experimentation (institutional and national) and with the Helsinki Declaration of 1975, as revised in 2000. All participants provided written informed consent and the study was approved by Nigerian Institute of Medical Research's Institutional Review Board.

\section{Consent for publication}

This manuscript does not contain any identifiable data in any form, either at the organizational level or at the individual level. 


\section{Author details}

'Department of Population Health, New York University School of Medicine, New York, NY, USA. ${ }^{2}$ College for Public Health \& Social Justice, Saint Louis University, Saint Louis, MO, USA. ${ }^{3}$ Nigerian Institute of Medical Research, Yaba, Lagos, Nigeria.

Received: 5 December 2019 Accepted: 10 June 2020

Published online: 26 June 2020

\section{References}

1. Freiberg MS, Chang C-CH, Kuller LH, Skanderson M, Lowy E, Kraemer KL, et al. HIV infection and the risk of acute myocardial infarction. JAMA Intern Med. 2013;173(8):614-22

2. Patel P, Rose CE, Collins PY, Nuche-Berenguer B, Sahasrabuddhe W, Peprah $E$, et al. Noncommunicable diseases among HIV-infected persons in lowincome and middle-income countries: a systematic review and metaanalysis. AIDS (London, England). 2018;32(Suppl 1):S5.

3. Peck RN, Shedafa R, Kalluvya S, Downs JA, Todd J, Suthanthiran M, et al. Hypertension, kidney disease, HIV and antiretroviral therapy among Tanzanian adults: a cross-sectional study. BMC Med. 2014;12(1):125.

4. Bigna JJR, Nansseu JRN, Um LN, Noumegni SRN, Simé PSD, Aminde LN, et al. Prevalence and incidence of pulmonary hypertension among HIVinfected people in Africa: a systematic review and meta-analysis. BMJ Open. 2016;6(8):e011921.

5. Forouzanfar MH, Liu P, Roth GA, Ng M, Biryukov S, Marczak L, et al. Global burden of hypertension and systolic blood pressure of at least 110 to 115 mm Hg, 1990-2015. Jama. 2017;317(2):165-82.

6. Piot P, Bartos M, Ghys PD, Walker N, Schwartländer B. The global impact of HIV/AIDS. Nature. 2001;410(6831):968.

7. Ogedegbe G, Plange-Rhule J, Gyamfi J, Chaplin W, Ntim M, Apusiga K, et al. Health insurance coverage with or without a nurse-led task shifting strategy for hypertension control: A pragmatic cluster randomized trial in Ghana. PLoS Med. 2018;15(5):e1002561.

8. Organization WH. World health statistics 2015: World Health Organization; 2015

9. Health NMo. Task-shifting and tasksharing policy for essential health care services in Nigeria. Nigeria: Nigerian Ministry of Health 2014.

10. Joshi R, Thrift AG, Smith C, Praveen D, Vedanthan R, Gyamfi J, et al. Taskshifting for cardiovascular risk factor management: lessons from the Global Alliance for Chronic Diseases. BMJ Glob Health. 2018;3(Suppl 3):e001092.

11. Callaghan M, Ford N, Schneider H. A systematic review of task-shifting for HIV treatment and care in Africa. Hum Resour Health. 2010;8(1):8.

12. Gyamfi J, Plange-Rhule J, Iwelunmor J, Lee D, Blackstone SR, Mitchell A, et al. Training nurses in task-shifting strategies for the management and control of hypertension in Ghana: a mixed-methods study. BMC Health Serv Res. 2017;17(1):104.

13. Limbani F, Thorogood M, Gómez-Olivé FX, Kabudula C, Goudge J. Task shifting to improve the provision of integrated chronic care: realist evaluation of a lay health worker intervention in rural South Africa. BMJ Glob Health. 2019;4(1):e001084.

14. Organization WH, Programme WHOCD. WHO CVD-risk management package for low-and medium-resource settings: World Health Organization; 2002.

15. El-Sadr WM, Goosby E. Building on the HIV platform: tackling the challenge of noncommunicable diseases among persons living with HIV. Aids. 2018; 32:S1-3.

16. Njuguna B, Vorkoper S, Patel P, Reid MJ, Vedanthan R, Pfaff C, et al. Models of integration of HIV and noncommunicable disease care in sub-Saharan Africa: lessons learned and evidence gaps. Aids. 2018;32:S33-42.

17. Iwelunmor J, Blackstone S, Gyamfi J, Airhihenbuwa C, Plange-Rhule J, Tayo $B$, et al. A concept mapping study of physicians' perceptions of factors influencing management and control of hypertension in sub-Saharan Africa. Int J Hypertens. 2015;2015.

18. Burke JG, O'Campo P, Peak GL, Gielen AC, McDonnell KA, Trochim WM. An introduction to concept mapping as a participatory public health research method. Qual Health Res. 2005;15(10):1392-410.

19. Trochim W, Kane M. Concept mapping: an introduction to structured conceptualization in health care. Int J Qual Health Care. 2005;17(3):187-91.

20. De Leeuw J, Mair P. Multidimensional scaling using majorization: SMACOF in $\mathrm{R} ; 2011$

21. Aarons GA, Sommerfeld DH, Chi BH, Ezeanolue EE, Sturke R, Guay L, et al. Concept mapping of PMTCT implementation challenges and solutions across 6 sub-Saharan African countries in the NIH-PEPFAR PMTCT implementation science alliance. JAIDS Journal of Acquired Immune Deficiency Syndromes. 2016;72:S202-S6.

22. Damschroder LJ, Aron DC, Keith RE, Kirsh SR, Alexander JA, Lowery JC. Fostering implementation of health services research findings into practice: a consolidated framework for advancing implementation science. Implement Sci. 2009;4(1):50.

23. Keith RE, Crosson JC, O'Malley AS, Cromp D, Taylor EF. Using the Consolidated Framework for Implementation Research (CFIR) to produce actionable findings: a rapid-cycle evaluation approach to improving implementation. Implement Sci. 2017;12(1):15.

24. Schell SF, Luke DA, Schooley MW, Elliott MB, Herbers SH, Mueller NB, et al. Public health program capacity for sustainability: a new framework. Implement Sci. 2013;8(1):15.

25. Michalski GV, Cousins JB. Differences in stakeholder perceptions about training evaluation: a concept mapping/pattern matching investigation Evaluation and Program Planning. 2000;23(2):211-30.

26. Blackstone S, Iwelunmor J, Plange-Rhule J, Gyamfi J, Quakyi NK, Ntim M, et al. Sustaining nurse-led task-shifting strategies for hypertension control: a concept mapping study to inform evidence-based practice. Worldviews Evid-Based Nurs. 2017;14(5):350-7.

27. Iwelunmor J, Ezechi O, Obiezu-Umeh C, Gbajabiamila T, Musa AZ, Oladele D, et al. Capabilities, opportunities and motivations for integrating evidencebased strategy for hypertension control into HIV clinics in Southwest Nigeria. PLoS One. 2019;14(6):e0217703.

28. Rosas SR, Kane M. Quality and rigor of the concept mapping methodology: a pooled study analysis. Evaluation and program planning. 2012;35(2):236-45.

29. Vorkoper S, Kupfer LE, Anand N, Patel P, Beecroft B, Tierney WM, et al. Building on the HIV chronic care platform to address noncommunicable diseases in sub-Saharan Africa: a research agenda. Aids. 2018;32:S107-S13.

30. Trochim WM. An introduction to concept mapping for planning and evaluation. Evaluation and program planning. 1989;12(1):1-16.

\section{Publisher's Note}

Springer Nature remains neutral with regard to jurisdictional claims in published maps and institutional affiliations.
Ready to submit your research? Choose BMC and benefit from:

- fast, convenient online submission

- thorough peer review by experienced researchers in your field

- rapid publication on acceptance

- support for research data, including large and complex data types

- gold Open Access which fosters wider collaboration and increased citations

- maximum visibility for your research: over $100 \mathrm{M}$ website views per year

At $\mathrm{BMC}$, research is always in progress.

Learn more biomedcentral.com/submissions 